\title{
Comparison of functional outcomes of arthroscopic ACL reconstruction using quadruple hamstring graft and quadriceps tendon grafts- A prospective study
}

\author{
Ashish Kumar', Sanjiv Kumar²*, Rahul Chauhan³, Deepak Kumar ${ }^{4}$ \\ ${ }^{1}$ Professor, ${ }^{2,4}$ Assistant Professor, ${ }^{3}$ Senior Resident, Dept. of Orthopaedics, KGMU, Lucknow, Uttar Pradesh, India
}

*Corresponding Author: Sanjiv Kumar

Email: sanjeevkumar98@gmail.com

\begin{abstract}
Introduction: Selection of graft for primary anterior cruciate ligament reconstruction have long been a topic of controversy. Among the vast range of graft options, hamstring autograft is most commonly used and quadriceps tendon autograft is less common.

Purpose: To compare the functional outcomes in quadriceps tendon graft and quadruple hamstring graft in primary anterior cruciate ligament reconstruction.

Materials and Methods: Thirty patients with post traumatic anterior cruciate ligament injury were taken into study. All 30 patients were randomised and two group were made. Group H Included 15 patients in which ACL reconstruction was done using quadruple hamstring graft and Group Q included 15 patients in which ACL reconstruction was done using quadriceps tendon graft. All patients were prospectively analysed. In post-operative period all patients were treated with standardized rehabilitation protocol and were evaluated at 6 weeks, 3 months and 6months by Lysholm knee score, quadriceps weakness, extension lag, quadriceps wasting, anterior drawer test, Lachman test and pivot shift test and compared.

Results: Mean difference in extensor lag between both groups was 1.07(p value 4.48), - 0.22(p value 3.59) and - 0.32(p value 2.28) at first, second and third follow-up but was not significant. Mean difference in quadriceps strength between both groups was 4.10 (p value 0.172 ), 2.04(p value 0.955 ) and -2.46 ( $\mathrm{p}$ value 0.218 ) at first, second and third follow-up but was not significant. Mean difference in quadriceps wasting between both groups was - 0.29(p value 0.380), - 0.33(p value 0.349) and - 0.26(p value 0.217) at first, second and third follow-up but was not significant. Mean difference in Lysholm knee score between both groups was 4.10(p value 0.172), 2.04(p value 0.955) and - 2.46 ( $p$ value 0.218 ) at first, second and third follow up but was not significant. No significant difference was found in both groups.

Conclusion: All the patients in whom ACL reconstruction were done showed good results irrespective of the choice of graft. The results showed no clinically significant difference at different follow-ups. Considering the small number of the study of short follow up, we recommend the study at larger scale for longer follow up to evaluate the results of both the procedures.
\end{abstract}

Keywords: Anterior collateral ligament reconstruction, Hamstring graft, Quadriceps graft.

\section{Introduction}

The goal of ACL surgery is to restore normal or almost normal stability in the knee and the level of function you had before the knee injury, limit loss of function in the knee, and prevent injury or degeneration to other knee structures. It is important to obtain the normal range of motion and regaining strength equal to uninjured leg. The application of knee arthroscopy to patient care has steadily expanded over the past two decades. Arthroscopy assisted techniques for ACL reconstruction have resulted in smaller incisions without disturbing adjacent uninvolved anatomic structures. The recovery and rehabilitation following these procedures has also been shortened. ${ }^{1}$

Graft options for anterior cruciate ligament reconstruction have always been a topic of controversy. Options include hamstring graft, quadriceps tendon graft and patellar tendon graft. Earlier patellar tendon used to be the graft of choice for primary ACL reconstruction, a place that has now been taken by hamstring graft. ${ }^{2}$ Though none of the graft material is ideal but hamstring graft is the closest owing to its low incidence of graft site morbidity, more strength and stiffness. Hamstring grafts have been in use for decades now giving us a lot of experience of it. ${ }^{3}$

The quadriceps tendon (QT) as a graft source for anterior cruciate ligament (ACL) and reconstruction has recently achieved increased attention. Although many knee surgeons have been using the QT as a graft for ACL revision surgery, it has never gained universal acceptance for primary ACL reconstruction. ${ }^{4}$ Quadriceps tendon has been in use since a long time but unlike before it has lately gained some popularity in primary ACL reconstruction due to its anatomic, histologic and biomechanical properties. ${ }^{5}$ In the late 1990s Stäubli et al. from Switzerland, published anatomic and biomechanical details of the QT and were the first advocates of its use as a primary ACL graft. Good clinical outcomes have been documented for the use of the QT in ACL surgery. ${ }^{6}$ The QT has also been successfully used for PCL reconstruction. The QT is a very versatile graft that can be harvested in different widths, thicknesses, and lengths. Quadriceps tendon grafts is less strong but can serve as an excellent graft option for revision ACL reconstruction where hamstring graft has already been taken or in persons whose hamstrings cannot be sacrificed. ${ }^{7}$ If done by minimally invasive technique it also gives smaller incisions and is easy to harvest as compared to hamstring graft. ${ }^{8}$ Studies have shown good clinical outcomes with low donor site morbidity. ${ }^{9}$

\section{Materials and Methods}

A prospective study conducted in patients admitted to the deptt. Of Orthopedic surgery at tertiary care centre over a period of 12 months (2016-2017) including 30 patients of anterior cruciate ligament injury secondary to post-trauma. All their demographic data were collected and documented at 
the time of their inclusion in the study. All 30 patients were randomised and two group were made. Group $\mathrm{H}$ Included 15 patients in which ACL reconstruction was done using quadruple hamstring graft and Group Q included 15 patients in which ACL reconstruction was done using quadriceps tendon graft. All Clinical / radiological proven cases of anterior cruciate ligament deficiency in active patients of age 18-45 who were symptomatic even after quadriceps strengthening exercise regime and brace application of adequate duration (4 weeks) with normal contra-lateral knee were included in this study. ACL reconstruction surgery was done once the acute inflammatory phase was over ie usually after 4-6 weeks of injury. The patients were allocated in both groups by using computer generated random number table. All the patients were informed about the study in detail and written informed consent was taken from each patient before surgery. Patients with other systemic diseases compromising their pre-anaesthetic fitness, or with associated fractures involving lower limbs or spine, or associated neurovascular injuries, patients with any other associated ligament injuries of the Knee (tear of posterior cruciate ligament, medial and lateral collateral ligament requiring treatment / with significant articular cartilage lesion exceeding grade III), patients with open physis, patients with significant arthritis (K-L grade III \& IV) or patients with local skin infections were not included in the study.

Patients were followed up post-operatively at 6 weeks, 3 months and 6 months. Post operatively functional status of all patients in both the groups were assessed by Lysholm knee score and by the extensor lag. Grading of laxity was evaluated by anterior drawer test, Lachman test and pivot shift test. Quadriceps muscle weakness evaluated by spring balance and quadriceps wasting was evaluated by measuring the quadriceps $15 \mathrm{Cm}$ proximal to joint line. Anterior drawer and Lachman tests are graded on the basis of anterior translation of tibia on femur as Grade +3 if the anterior translation of tibia is more than $10 \mathrm{~mm}$, Grade +2 if anterior translation of tibia is in between $5-10 \mathrm{~mm}$, and Grade +1 if anterior translation of tibia is less than $5 \mathrm{~mm}$. The pivot shift test is clinically graded as following Grade I -Gentle twisting slide with tibia twisting internally maximally, Grade II -Clunk with tibia neutral, negative when tibia externally rotated, Grade III -Painless glide for examiner and patient, Grade IV - Jamming and Plowing impingement. Knee extension lag was present if extension lag is more than $5^{0}$ and considered absent if this was less than $5^{\circ}$. Similarly, Quadriceps muscle weakness was measured at $90^{\circ}$ knee flexion with the help of spring balance and was interpreted as present if power was less than $85 \%$ of uninvolved lower limb or absent if power was more than $85 \%$ of uninvolved lower limb.

\section{Surgical Technique}

Part painted and draped and tourniquet deflated. Standard anterolateral and anteromedial portals made. Diagnostic arthroscopy done. After confirming the ACL tear notch plasty was done. Except for the graft harvesting and graft preparation rest of the surgical steps were same for both the groups and ACL reconstruction was done in standard manner. On the femoral side variable loop endo button and on tibial side interference screw were used for graft fixation in both the groups.

\section{Quadruple Hamstring Graft}

A $2 \mathrm{Cm}$ incision given $2 \mathrm{Cm}$ medial to tibial tuberosity. Subcutaneous tissue bluntly dissected and hamstrings identified. Any soft tissue slips attached to tendon divided and tendon harvested inside out using an open stripper. Both semitendinosus and gracilisused if graft from semitendinosus found to be of inadequate thickness $(<8 \mathrm{~mm})$. Graft quadrupled using Krackow sutures and attached to endobutton-tight rope. ${ }^{8}$ (Fig. 2)

\section{Quadriceps Tendon Graft}

The quadriceps tendon graft was harvested by an open technique, requiring a 6 to $8 \mathrm{~cm}$ longitudinal incision. Mid $1 / 3^{\text {rd }}$ portion of quadriceps muscle was selected and marked and desired depth and length of tendon marked precisely with marker using ruler. Quadriceps tendon separated from its base using scalpel in distal to proximal direction. Desired length of quadriceps tendon cut. Krackow sutures applied using fiber tape with fibre wire and on the other end a variable tight rope with endo-button attached. ${ }^{4}$ (Fig. 1)
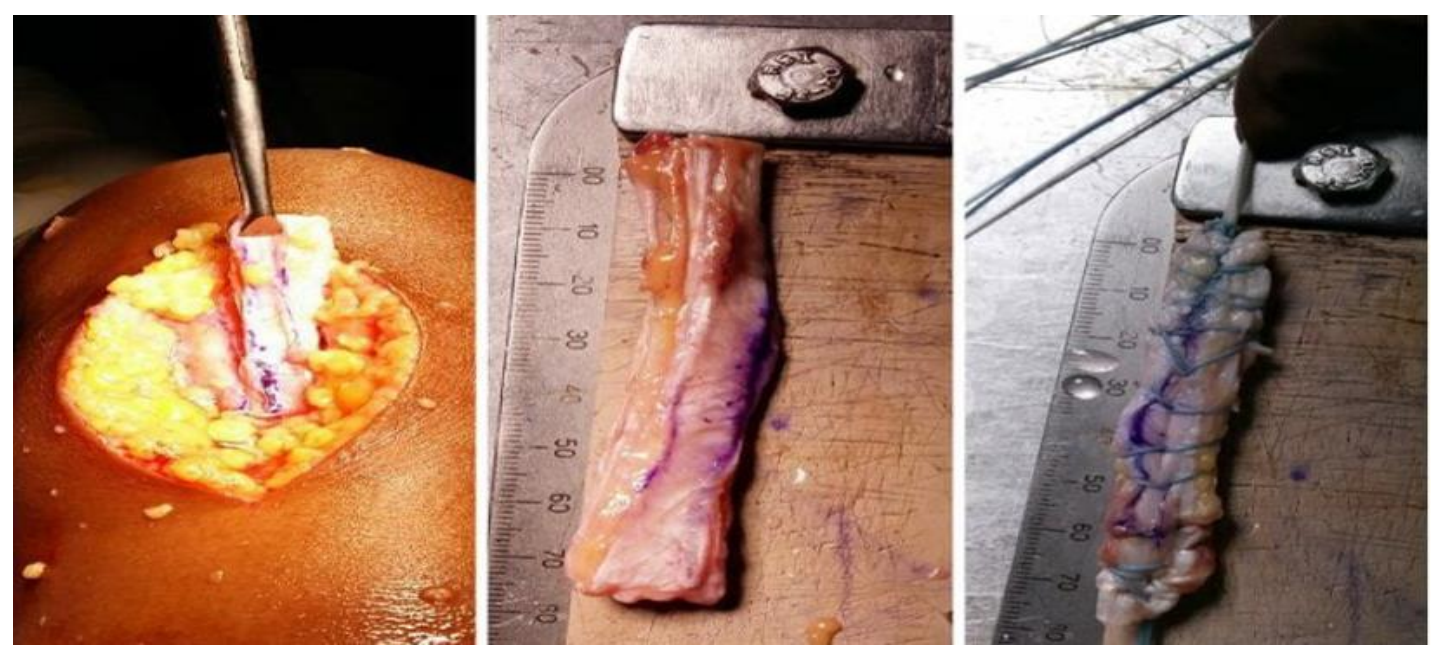

Fig. 1: Showing harvesting of quadriceps tendon graft, its size and final preparation of the graft 


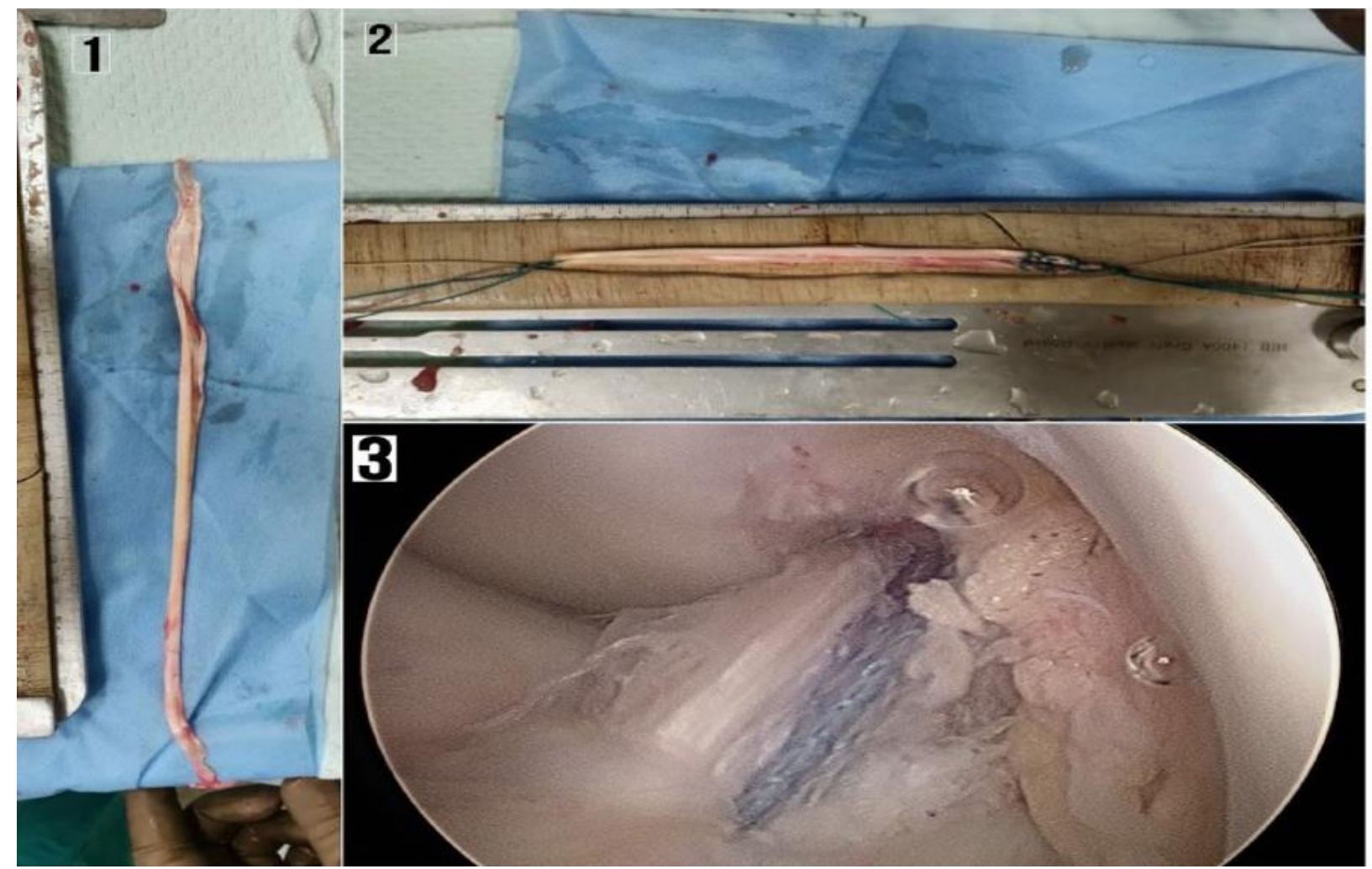

Fig. 2: Showing Hamstring graft, its preparation and arthroscopic view after final fixation

\section{Results}

Present study included 30 patients of comparable demography in two groups having 15 patients each. Postoperatively, all patients were assessed for extension lag, quadriceps wasting, quadriceps strength, and Lysholm knee score at 6 weeks, 3 months and 6 months.(Table 1) Mean difference in extensor lag between both groups was $1.07(\mathrm{p}$ value 4.48 ), -0.22 (p value 3.59 ) and -0.32 (p value 2.28 ) at first, second and third follow up but was not significant.
Mean difference in quadriceps strength between both groups was 4.10 (p value 0.172$), 2.04(\mathrm{p}$ value 0.955$)$ and $-2.46(\mathrm{p}$ value 0.218 ) at first, second and third follow-up but was not significant. Mean difference in quadriceps wasting between both groups was -0.29 ( $p$ value 0.380 ), -0.33 ( $p$ value 0.349 ) and -0.26 ( $\mathrm{p}$ value 0.217 ) at first, second and third follow-up but was not significant. Mean difference in Lysholm knee score between both groups was 4.10 (p value 0.172$), 2.04(\mathrm{p}$ value 0.955$)$ and $-2.46(\mathrm{p}$ value 0.218$)$ at first, second and third follow-up but was not significant. No significant difference was found in both groups.

Table 1

\begin{tabular}{|c|c|c|c|c|c|c|c|c|c|c|c|c|}
\hline & \multicolumn{3}{|c|}{ Extension lag (degree) } & \multicolumn{3}{|c|}{ Quadriceps wasting (cm) } & \multicolumn{3}{|c|}{$\begin{array}{c}\text { Quadriceps strength (as } \\
\text { per spring balance@ @ } 90^{\circ} \text { of } \\
\text { knee flexion) }\end{array}$} & \multicolumn{3}{|c|}{ Lysholm knee score } \\
\hline & Group H & $\begin{array}{c}\text { Group } \\
\text { Q }\end{array}$ & $\begin{array}{c}\text { Mean } \\
\text { Difference }\end{array}$ & $\begin{array}{c}\text { Group } \\
\mathrm{H}\end{array}$ & $\begin{array}{c}\text { Group } \\
\text { Q }\end{array}$ & $\begin{array}{c}\text { Mean } \\
\text { Difference }\end{array}$ & $\begin{array}{c}\text { Group } \\
\mathrm{H}\end{array}$ & $\begin{array}{c}\text { Group } \\
\text { Q }\end{array}$ & \begin{tabular}{|c|} 
Mean \\
Difference \\
\end{tabular} & $\begin{array}{c}\text { Group } \\
\mathrm{H}\end{array}$ & $\begin{array}{c}\text { Group } \\
\text { Q }\end{array}$ & $\begin{array}{c}\text { Mean } \\
\text { Difference }\end{array}$ \\
\hline \begin{tabular}{|l|} 
Mean \\
6 weeks
\end{tabular} & 11.57 & 10.5 & $\begin{array}{c}1.07 \\
\text { (p value } \\
4.48)\end{array}$ & 2.41 & 2.7 & & 27.25 & 26.59 & $\begin{array}{c}4.10(\mathrm{p} \\
\text { value } \\
0.172)\end{array}$ & 51.10 & 47 & $\begin{array}{l}4.10(\mathrm{p} \\
\text { value } \\
0.172)\end{array}$ \\
\hline 3 months & 5.78 & 6.0 & $\begin{array}{c}0.22 \\
(\mathrm{p} \text { value } \\
3.59)\end{array}$ & 1.52 & 1.85 & $\begin{array}{c}0.33 \\
\text { (p value- } \\
0.349)\end{array}$ & 46.10 & 56.09 & $\begin{array}{l}2.04(\mathrm{p} \\
\text { value } \\
0.955)\end{array}$ & 72.04 & 70 & $\begin{array}{l}2.04(\mathrm{p} \\
\text { value } \\
0.955)\end{array}$ \\
\hline 6 months & 1.18 & 1.5 & $\begin{array}{c}0.32 \\
\text { (p value } \\
2.28) \\
\end{array}$ & 0.49 & .75 & $\begin{array}{l}0.26(\mathrm{p} \\
\text { value } \\
0.217)\end{array}$ & 88.73 & 90.85 & $\begin{array}{l}2.46(\mathrm{p} \\
\text { value } \\
0.218)\end{array}$ & 87.97 & 90.40 & $\begin{array}{l}2.46(\mathrm{p} \\
\text { value } \\
0.218)\end{array}$ \\
\hline Intragroup & 10.39 & 9 & & 1.92 & 1.95 & & 61.48 & 64.26 & & 36.87 & 43.4 & \\
\hline
\end{tabular}




\section{Discussion}

ACL reconstruction first described in 1917 by Hey-Groves using iliotibial band autograft, since then surgeons have been looking for an ideal graft for ACL reconstruction. An ideal graft should have structural and biomechanical properties similar to native ACL, allow secure fixation and rapid biological incorporation and have limited donor site morbidity. Since its advent in 1963, BPTB graft has been widely used in the primary surgical reconstruction of the ACL due to its strength, stiffness and potential for bone integration (due to the presence of bone plugs at its ends). ${ }^{10}$

Biomechanical studies on cadavers have shown that the middle third of the BPTB graft has an initial strength and stiffness comparable to, or maybe even greater than, those of the native ACL. There are many post-operative complications related to donor site morbidity, such as: patellar fractures, weakening of the quadriceps muscles, patellar tendon rupture and patellar tendonitis which lead to decrease use of this graft in present era. ${ }^{11,12}$

To overcome the problems associated with BPTB graft morbidity, many surgeons have started, systematically, to use the tendons of the gracile and semitendinosus muscles (hamstrings). The advantages of this choice are: a greater cross-sectional area and maintenance of the integrity of the extensor mechanism. Furthermore, tensile strength of the quadrupled HT graft is nearly three times greater than that of the normal ACL. ${ }^{13}$

The disadvantages of HT graft may include a longer healing time and graft integration time within the bone tunnel because of the absence of bone plugs at the ends of the graft. Moreover, the lack of both hamstrings eliminates the protective and stabilizing action that these muscles exert on the knee during specific movements; this condition predisposes to rupture of the ACL graft: the hamstrings and ACL together create a reflex-arc that contributes to proprioceptive control. ${ }^{14}$

The first use of the quadriceps tendon (QT) graft dates back to 1979. However, its poor biomechanical strength and the unsatisfactory clinical outcomes meant that this choice remained unpopular throughout the 1980s. Its theoretical advantages include the fact that the harvesting does not affect the patellar tendon and thus reduce the risk of intra-patellar scarring. Also, there is no risk of injury to the infra-patellar branch of the saphenous nerve, which is a common complication during PT harvesting. ${ }^{15,16}$

A graft used for surgical ACL reconstruction should be one that, as far as possible, recreates the anatomical and biomechanical properties of the native ligament, that guarantees safe fixation, and that provides rapid biological integration, reducing recovery time and donor site morbidity. ${ }^{17}$

There are very few comparative studies of ACL reconstruction using hamstrig graft and quadriceps tendon graft are available in literature considering the results in terms of quadriceps wasting, quadriceps strength, Lysholm knee score and extension lag with long term follow up. In the study of Schulz et al, mean Lysholm knee score was 89 points at 2 to 3 year follow up. Kohl et al in their study had a mean
Lysholm knee score of 94. In our study mean lysholm knee score in hamstring graft group and quadriceps tendon group was 87.94 and 90.40 at 6 months follow up respectively. ${ }^{9,18}$

Schulz et al in their study had only $18.2 \%$ grade 2 lachman test at 2-3 years followup rest all had grade 1 and grade 0 lachman test. Kim at al in their study had $4.61 \%$ patients with hamstring graft showing grade 2 lachman test at 2 years while all others had a grade 0 or 1 lachman test. In our study $11.8 \%$ and $0 \%$ patients had grade 2 lachman test at 6 months follow up in hamstring graft group and quadriceps tendon group. ${ }^{18,19}$ Schulz et al in their study had no patients with positive pivot shift test. Kim at al in their study had $4.61 \%$ patients with hamstring graft showing grade 2 pivot shift test at 2 years while all others had a grade 0 or 1 pivot shift test. In our study none of the patients in any group showed positive pivot shift test after last follow up..$^{10,11}$

\section{Conclusion}

All the patients in whom ACL reconstruction were done showed good results irrespective of the choice of graft. The results showed no clinically significant difference at different follow-ups. Although use of Hamstring graft for ACL reconstruction is standard material of choice for primary ACL reconstruction, quadriceps graft can be of choice for ACL reconstruction in revision surgeries. Considering the small number of the study of short follow up, we recommend the study at larger scale for longer follow up to evaluate the results of both the procedures.

\section{Conflict of Interest: None.}

\section{References}

1. Cosimo Tudisco, Salvatore Bisicchia, "drilling the femoral tunnel during ACL reconstruction: Transtibial vs. Anteromedial portal techniques" Orthopedics. 2012;35(8):e1166-e1172.

2. Lalith Mohan Chodavarapu, Asif Hussain K. S., K. K. Kiran Kumar, Chandrasekhar Patnala, Harikrishna Yadoji. Analysis of functional outcome of anterior cruciate ligament reconstruction using quadruple hamstring graft. 2017

3. Alec A. Macaulay, Dean C. Perfetti, William N. Levine, Anterior Cruciate Ligament Graft Choices. 2012.

4. Christian Fink, Mirco Herbort, Elisabeth Abermann, and Christian Hoser, Minimally Invasive Harvest of a Quadriceps Tendon Graft With or Without a Bone Block. 2014

5. Joseph P. DeAngelis, John P. Fulkerson. Quadriceps TendonA Reliable Alternative for Reconstruction of the Anterior Cruciate Ligament. 2007 Oct.

6. Hyuk Soo Han, Sang Cheol Seong, Sahnghoon Lee Anterior Cruciate Ligament Reconstruction.

7. Giuliano Cerulli, Giacomo Placella, Enrico Sebastiani, Matteo Maria Tei, Andrea Speziali, and Francesco Manfreda ACL Reconstruction: Choosing the Graft Joints. 2013;1(1):18-24.

8. Cavaignac E, Coulin B, Tscholl P, Nik Mohd Fatmy N, Duthon V, Menetrey J Is Quadriceps Tendon Autograft a Better Choice Than Hamstring Autograft for Anterior Cruciate Ligament Reconstruction? A Comparative Study with a Mean Follow-up of 3.6 Years. Am J Sports Med 2017;45(6):1326-32.

9. Kohl S, Stutz C, Decker S, Ziebarth K, Slongo T. Mid-term results of transphyseal anterior cruciate ligament reconstruction in children and adolescents. Knee 2014;21(1):80-5. 
10. Jones KG. Reconstruction of the anterior cruciate ligament. A technique using the central one-third of the patellar ligament. $J$ Bone Joint Surg 1963;45A:925-932.

11. Almekinders LC, Moore T, Freedman D. Post-operative problems following anterior cruciate ligament reconstruction. Knee Surg Sports Traumatol Arthrosc 1995;3:78-82.

12. Marder RA, Raskind JR, Carroll M. Prospective evaluation of arthroscopically assisted anterior cruciate ligament reconstruction. Patellar tendon versus semitendinosus and Gracilis tendons. Am J Sports Med 1991;19:478-84.

13. Brown $\mathrm{CH}$, Jr, Steiner ME, Carson EW. The use of hamstring tendons for anterior cruciate ligament reconstruction. Technique and results. Clin Sports Med 1993;12:723-56.

14. Cerulli G, Benoit DL, Lamontagne M. In vivo anterior cruciate ligament strain behaviour during a rapid deceleration movement: case report. Knee Surg Sports Traumatol Arthrosc 2003;11:307-11.

15. Marshall JL, Warren RF, Wickiewicz TL, et al. The anterior cruciate ligament: a technique of repair and reconstruction. Clin Orthop 1979;143:97-106.

16. Staubli HU, Schatzmann L, Brunner P. Mechanical tensile properties of the quadriceps tendon and patellar ligament in young adults. Am J Sports Med 1999;27:27-34.
17. Giuliano Cerulli, Giacomo Placella, Enrico Sebastiani, Matteo Maria Tei, Andrea Speziali, andFrancesco Manfreda. ACL Reconstruction: Choosing the Graft. Joints 2013;1(1):18-24.

18. Arndt P Schulz, Vivien Lange, Justus Gille, Christine Voigt, Susanne Fröhlich, Markus Stuhr, and Christian Jürgens Anterior cruciate ligament reconstruction using bone plug-free quadriceps tendon autograft: intermediate-term clinical outcome after 24-36 months Open Access. J Sports Med 2013;4:243-9.

19. Sung-Jae Kim, Su-Keon Lee, Chong Hyuk Choi, Sung-Hwan Kim Graft Selection in Anterior Cruciate Ligament Reconstruction for Smoking Patients. Am J Sports Med 2014;42:166.

How to cite this article: Kumar S, Kumar A, Chauhan R, Kumar D. Comparison of functional outcomes of arthroscopic ACL reconstruction using quadruple hamstring graft and quadriceps tendon grafts- A prospective study. Indian J Orthop Surg 2019;5(2):149-53. 